International Journal of Pure and Applied Mathematics

Volume 103 No. 4 2015, 683-695

ISSN: 1311-8080 (printed version); ISSN: 1314-3395 (on-line version)

url: http://www.ijpam.eu

doi: http://dx.doi.org/10.12732/ijpam.v103i4.8

ijpam.eu

\title{
OBSERVATIONS ON SOME CLASSES OF ALGEBRAS
}

\author{
Mehrzad Ahmadi Abnavi ${ }^{1}$, Mohammad Naghshinehfard ${ }^{2} \S$ \\ ${ }^{1}$ Department of Mathematics \\ Shiraz Branch \\ Islamic Azad University \\ Shiraz, IRAN \\ ${ }^{2}$ Department of Mathematics \\ Jahrom Branch \\ Islamic Azad University \\ Jahrom, IRAN
}

\begin{abstract}
In this paper we investigate some analouge properties of finite p-groups to nilpotent lie algebras. In particular we show that there is a relation between nilpotency class of subalgebras and origin lie algebra itself.
\end{abstract}

AMS Subject Classification: $17 \mathrm{~B} 12$

Key Words: nilpotency class

\section{Introduction}

A classical problem in finite p-groups concerns the nilpotency class of groups in which every proper subgroup is nilpotent of a given class bounded by a fixed positive integer. Macdonald in [4] has made a substantial contribution in this area. The aim of this paper is to study the analogous problem for finitedimensional nilpotent Lie algebras. We study the structure of Lie algebras in

Received: June 18, 2015

(c) 2015 Academic Publications, Ltd.

${ }^{\S}$ Correspondence author url: www.acadpubl.eu 
terms of their nilpotency class by putting certain conditions on every maximum subalgebra or every proper subalgebra. We have used Macdonald's work as a guide. We can categorize our findings in three ways. some results carry over with little change, some results carry over with modifications and simplifications in the proofs, and some results are different than the results in groups. Before the results are shown, we begin with the following facts and notations.

\section{Preliminary Notes}

Let $x_{1}, x_{2}, \ldots, x_{n}$ be elements in Lie algebra L. The commutator $\left[x_{1}, x_{2}, \ldots, x_{n}\right]$ in $\mathrm{L}$ is defined as

$$
\left[x_{1}, x_{2}, \ldots, x_{n}\right]=\left[\left[x_{1}, x_{2}, \ldots, x_{n-1}\right], x_{n}\right]
$$

for all $n \geq 2$. Moreover, we define

$$
\left[x_{1}, x_{2}, \ldots, x_{n} ; y_{1}, y_{2}, \ldots, y_{m}\right]=\left[\left[x_{1}, x_{2}, \ldots, x_{n}\right],\left[y_{1}, y_{2}, \ldots, y_{m}\right]\right]
$$

for any integers $\mathrm{m}$ and $\mathrm{n}$. We also denote $\langle x\rangle$ and $(\mathrm{x})$ as an ideal generated by $\mathrm{x}$ and a subalgebra generated by $\mathrm{x}$, respectively. The following definitions follow from [4].

Let $\mathrm{x}$ be an element in a Lie algebra $\mathrm{L}$ for which $[x, \overbrace{y, \ldots, y}^{n}]=0$ for all $y \in L$. Then $\mathrm{x}$ is said to be an nth Engel element. Furthermore, if every element of $\mathrm{L}$ has this property, than L is said to satisfy the nth Engel condition.

Definition 1. Let $\mathrm{L}$ be a Lie algebra. The series of ideals

$$
L \supseteq L^{2} \supseteq L^{3} \supseteq \cdots
$$

is called the lower central series of $\mathrm{L}$ where $L^{2}=[L, L]$ and $L^{m}=\left[L, L^{m-1}\right]$ for $\mathrm{m}=3,4, \ldots$

Definition 2. A Lie algebra $L$ is said to be nilpotent if $L^{m+1}=0$ for some positive integer $\mathrm{m}$. If also $L^{m} \neq 0$, then we say that $\mathrm{L}$ has class precisely $\mathrm{m}$.

Definition 3. A Lie algebra $L$ is said to be metabelian if $L^{2}$ is abelian.

Lemma 4. Let $L$ be a metabelian Lie algebra, then $[u, v, x, y]=[u, v, y, x]$ for $x, y, u, v \in L$.

Proof. Since L is metabelian, using the Jacobi identity, we obtain

$$
0=[u, v ; x, y]=[u, v, x, y]+[x ;, u, v, y]=[u, v, x, y]-[u, v, y, x]
$$




$$
[u, v, x, y]=[u, v, y, x] .
$$

Furthermore, the value of $\left[x_{1}, x_{2}, x_{3}, \ldots, x_{n}\right]$ is unaltered when $x_{3}, \ldots, x_{n}$ are permuted in any way.

Lemma 5. For all elements $x \in L$, suppose that $\langle x\rangle$ has class n.Then $L$ satisfies the $(n+1)$ th Engel condition.

Proof. Since $\mathrm{x}$, and $[x, y] \in\langle x\rangle$, the commutator

$$
[[x, y], \underbrace{x, \ldots, x}_{n}]=0 .
$$

Therefore, y satisfies the $(\mathrm{n}+1)$ th Engel condition for all $y \in L$. As a result, $\mathrm{L}$ satisfies the $(\mathrm{n}+1)$ th Engel condition.

\section{A Bound on the Nilpotency Class}

The following lemma is well known, see [2].

Lemma 6. Suppose $M$ and $N$ are nilpotent ideals of a Lie algebra $L$. If $M$ and $N$ have class $k$ and $l$, respectively, then $M+N$ has class at most $k+l$.

The following lemma is similar to a group theoretic result in [3].

Lemma 7. For every Lie algebra, the commutator of the form $\left[x_{1}, x_{2}, \ldots, x_{n}\right.$, $y]$ can be expressed as the sum of $2^{n-1}$ commutators of the form $\pm\left[y, x_{\pi(1)}, \ldots\right.$, $\left.x_{\pi(n)}\right]$, where $\pi \in S_{n}$.

Proof. We shall use induction to prove this lemma. When $\mathrm{n}=1$, we have

$$
[x, y]=-[y, x] .
$$

Assume true for a fixed positive integer $n-1$; that is

$$
\left[x_{1}, \ldots, x_{n-1}, y\right]= \pm \sum_{j=1}^{2^{\mathrm{n}-1}}\left[y, x_{\pi_{\mathrm{j}}(1)}, \ldots, x_{\pi_{\mathrm{j}}(n-1)}\right]
$$

Then,we shall show that it is also true in the case of $\left[x_{1}, \ldots, x_{n}, y\right]$.For convenience, let $\left[x_{1}, \ldots, x_{n-1}\right]=\mathrm{z}$. The Jacobi identity gives us

$$
\left[z, x_{n}, y\right]=\left[z, y, x_{n}\right]-\left[z ; y, x_{n}\right] .
$$


Consider each commutator on the right-hand side separately. By induction hypothesis and Eq. (1), we have

$$
[z, y]= \pm \sum_{j=1}^{2^{\mathrm{n}-2}}\left[y, x_{\pi_{\mathrm{j}}(1)}, \ldots, x_{\pi_{\mathrm{j}}(n-1)}\right]
$$

and

$$
\begin{gathered}
{\left[z, y, x_{n}\right]= \pm\left[\sum_{j=1}^{2^{\mathrm{n}-2}}\left[y, x_{\pi_{\mathrm{j}}(1)}, \ldots, x_{\pi_{\mathrm{j}}(n-1)}\right], x_{n}\right]} \\
= \pm \sum_{j=1}^{2^{\mathrm{n}-2}}\left[y, x_{\pi_{\mathrm{j}}(1)}, \ldots, x_{\pi_{\mathrm{j}}(n-1)}, x_{n}\right]
\end{gathered}
$$

Similarly

$$
-\left[z ; y, x_{n}\right]= \pm \sum_{j=1}^{2^{\mathrm{n}-1}}\left[y, x_{n}, x_{\pi_{\mathrm{j}}(1)}, \ldots, x_{\pi_{\mathrm{j}}(n-1)}\right]
$$

Thus

$$
\left[z, x_{n}, y\right]= \pm \sum_{j=1}^{2^{\mathrm{n}-1}}\left[y, x_{\pi_{\mathrm{j}}(1)}, \ldots, x_{\pi_{\mathrm{j}}(n)}\right]
$$

Therefore, $\left[x_{1}, \ldots, x_{n}, y\right]$ is the sum of $2^{n-1}$ commutators of the form

$$
\left[y . x_{\pi(1)}, \ldots, x_{\pi(n)}\right]
$$

where $\pi \in S_{n}$.

\section{Metabelian Nilpotent Lie Algebras}

The emphasis of this section concerns the class of finite-dimensional nilpotent metabelian Lie algebras. We shall first look at the case of the Lie algebra in which every proper subalgebra has nilpotency class bounded by a fixed number $n$. Since every maximum subalgebra of a nilpotent Lie algebra is an ideal [1], if every proper subalgebra has class at most $n$, then the class of the Lie algebra is at most $2 n$ by Lemma 7 . We shall further address the question of when the class of a nilpotent Lie algebra is precisely $2 n$. 


\subsection{Nilpotency Class of Metabelian Lie Algebras}

Theorem 8. Let $L$ be a finite-dimensional metabelian Lie algebra over a field $F$ and suppose that $L$ has class precisely $2 n$ with every proper subalgebra of class at most $n$. Then

(1) If $F$ has characteristic 0 , then $n=1$.

(2) If $F$ has characteristic $p$, where $p$ is a prime number and $n \neq p^{\alpha}(\alpha \in$ $F)$, then $n=1$.

Before we prove above theorem, we gather elementary facts.

Lemma 9. Let $L$ be a finite-dimensional Lie algebra in which every maximal subalgebra has class at most $n$. Then

(1) $\langle x\rangle$, the ideal generated by $\mathrm{x}$, has class at most $\mathrm{n}$ for all elements $\mathrm{x}$ in $L$, and $L$ satisfies the $(n+1)$ th Engel condition.

(2) If $L$ has class precisely $2 n$, then two elements generate $L$.

Proof. (1) If $\langle x\rangle$ is proper then it has class at most $\mathrm{n}$ and $\mathrm{L}$ satisfies the $(n+$ 1)th Engel condition. So suppose that $L=\langle x\rangle=(x)+L^{2}$. Then $\operatorname{dim}\left(L / L^{2}\right)=$ 1 , whence $\operatorname{dim}(L)=1$.

(2) Suppose L has class precisely $2 n$, and let $\mathrm{M}$ be a maximal subalgebra of L. $\mathrm{M}$ is also an ideal in $\mathrm{L}$ because $\mathrm{L}$ is nilpotent. We choose an arbitrary $x \in L$ such that $x \notin M$, then $L=\langle x\rangle+$ M. By Lemma 7 , the ideal $\langle x\rangle$ has class precisely $\mathrm{n}$. Observe that any commutator element in $\langle x\rangle$ looks like $\left[x, y_{1}, \ldots, y_{t}\right]$ where $t \geq 0$.For each $t_{i} \geq 0$, we consider

$$
[\underbrace{\left[x, y_{11}, \ldots, y_{1 t_{1}}\right], \ldots,\left[x, y_{n 1}, \ldots, y_{n t_{n}}\right]}_{n}] \neq 0 .
$$

It follows that

$$
[\underbrace{\left[\left[x, y_{11}, \ldots, y_{1 t-1_{1}}\right], y_{1 t_{1}}\right],\left[x, y_{21}, \ldots, y_{2 t_{2}}\right], \ldots\left[x, y_{n 1}, \ldots, y_{n t_{n}}\right]}_{n+1} \neq 0
$$

Because of $\left\langle x, y_{1 t_{1}}\right\rangle$ has class greater than $\mathrm{n}$, we can conclude that

$$
\left\langle x, y_{1 t_{1}}\right\rangle=L .
$$

This complete the proof of this lemma; we are now realy to prove theorem 9 in which we rely heavily on Lemma 5 . 


\subsection{A Bound on the Nilpotency Class}

Proof. In accordance with previous lemma, let $\langle a, b\rangle=L$, and consider a commutator of length $2 n$ of the form

$$
\left[x_{1}, x_{2}, \ldots, x_{2 n}\right]=0
$$

whenever $\mathrm{n}+1$ of $x_{i}$ are equal because of the $(\mathrm{n}+1)$ th Engel condition. suppose $n \geq 1$, and that $\mathrm{L}$ has class precisely $2 \mathrm{n}$. We denote

$$
\begin{gathered}
x_{1}=a, \quad x_{2}=x_{3}=\ldots=x_{n+2}=a+b \\
x_{n+3}=\ldots=x_{n+r+2}=a, \\
x_{n+r+3}=\ldots=x_{2 n}=b,
\end{gathered}
$$

where $0 \leq r \leq n-2$. We then have

$$
\overbrace{[a, \underbrace{a+b, \ldots a+b}_{n+1}, \underbrace{a, \ldots, a, b, \ldots, b}_{n-2}]}^{2 n}=0
$$

Because there are $\mathrm{n}+1$ terms of the $(a+b)^{\prime} s$. Any product in $\mathrm{L}$ is the sum of the products of commutators involving only a and b,and these can be left normed. It suffices to show that the left normed product of $2 \mathrm{n}$ terms, each of which is a or b,is 0 .

The expansion of Eq. (2) gives some commutators that contain at least $\mathrm{n}+1$ of $a^{\prime} s$ and other commutators that contain at least $\mathrm{n}+1$ of $b^{\prime} s$. These are 0 by the Engel condition. Thus, we only have to consider commutators that contain $\mathrm{n}$ of $a^{\prime} s$ and $\mathrm{n}$ of $b^{\prime} s$. Then we are going to count the number of those commutators. Observe that the choices of commutators of length $2 \mathrm{n}$ with $\mathrm{n}$ of $a^{\prime} s$ and n of $b^{\prime} s$ are:

$$
\begin{gathered}
\left(\begin{array}{l}
n \\
1
\end{array}\right)[a, b, a, \underbrace{b, \ldots, b}_{n-1}, \underbrace{a, a, \ldots, a}-n-2]=0 \\
\left(\begin{array}{c}
n \\
2
\end{array}\right)[a, b, a, a, \underbrace{b, \ldots, b}_{n-1}, \underbrace{a, \ldots, a}_{n-3}]=0 \\
\left(\begin{array}{c}
n \\
n-1
\end{array}\right)[a, b, \underbrace{a, \ldots, a}_{n-1}, b, \underbrace{b, b, \ldots, b}_{n-2}]=0
\end{gathered}
$$


Since L is metabelian, we can permute each commutator above and put it in the form of

$$
[a, b, \underbrace{a, \ldots, a}_{n-1}, \underbrace{b, \ldots, b}_{n-1}] .
$$

Denote

$$
c=[a, b, \underbrace{a, \ldots, a}_{n-1}, \underbrace{b, \ldots, b}_{n-1}]
$$

and obtain the following cases:

Case 1. When $\operatorname{char}(F)=0$.

We then have $\left(\begin{array}{c}n \\ i\end{array}\right) c=0$

Case 2. When char $(F)=p$ and $n \neq p^{\alpha}$. The greatest common divisor of $\left(\begin{array}{l}n \\ 1\end{array}\right), \ldots,(n-1)$ is 1 . Thus,

$$
\sum_{n=1}^{n-1} \alpha_{i}\left(\begin{array}{c}
n \\
i
\end{array}\right)=1
$$

for some integers $\alpha_{i}$. Consequently, we multiply the above equation by $\mathrm{c}$ on each side yields that $\mathrm{c}=0$.

From the preceding two cases above, a commutator of length $2 \mathrm{n}$ in L equals 0 . $\mathrm{L}$ has class at most $2 \mathrm{n}-1$ which is a contradiction. Thereby, $\mathrm{n}$ must be 1 .

Note that the result of $n=1$ holds in the case of finite p-groups as well.Next, we move to consider the nilpotency class in the case of a metabelian Lie algebra when $\mathrm{n}$ is a power of a prime number $\mathrm{p}$. Unlike the two cases previously mentioned in theorem 9, we attempt to show that $\mathrm{n}$ does not have to be 1 , which is different than the result originally derived by Macdonald in groups.

We remark that the result of $\mathrm{n}$ greater than or equal to 1 in the case of $n=p^{\alpha}$ does not hold true in groups; $\mathrm{n}$ is always 1 when dealing with finite p-groups regardless of the relationship between $\mathrm{n}$ and $\mathrm{p}$.

\section{Nilpotency of Non-Metabelian Lie Algebras}

In this section, we relax the metabelian assumption. Without the metabelian property, the problem for determining the nilpotency class of a Lie algebra becomes more difficult. 


\subsection{The General Theorems}

We wish to determine a bound on the class of a given nilpotent Lie algebra, provided that we know about the class of the given subalgebras. In the next theorem, all subalgebras of dimension $r$ have class at most $n$, and $m$ denotes the difference between the dimension of the Lie algebra and $r$.

Theorem 10. Let $L$ be a Lie algebra of dimension $r+m$ and class not egual to $n$. Suppose each subalgebra of dimension $r$ has class at most $n$. Then $L$ has a set of $m+n$ elements which generates $L$.

Proof. Suppose that each set of generators of $\mathrm{L}$ contains at least $\mathrm{m}+\mathrm{n}+1$ elements.

Let $x_{1}, x_{2}, \ldots, x_{n+1}$ be arbitrary elements in L.Suppose

$$
H_{n+1}=\left\langle x_{1}, x_{2}, \ldots, x_{n+1}\right\rangle
$$

and $\operatorname{dim}\left(H_{n+1}\right) \geq r+1$.Form a strictly increasing chain

$$
H_{n+1} \subset H_{n+2} \subset \ldots \subset L
$$

by adjoining one additional element at a time.

If $\operatorname{dim}\left(H_{n+1}\right) \geq r+1$, then $\operatorname{dim}\left(H_{n+1}\right) \geq r+t$ for any integer t. Observe that $H_{n+m}=L$, and $\mathrm{L}$ is generated by $\mathrm{n}+\mathrm{m}$ elements; which is a contradiction. Therefore, $\operatorname{dim}\left(H_{n+1}\right)$ must be less than or equal to $\mathrm{r}$; that is $\operatorname{dim}\left(H_{n+1}\right) \leq r$ for any $\mathrm{n}+1$ elements in L. Hence

$$
H_{n+1}=\left\langle x_{1}, x_{2}, \ldots, x_{n+1}\right\rangle
$$

has class at most $\mathrm{n}$ by assumption. Since each $x_{i}$ is arbitrary chosen, the product of any $\mathrm{n}+1$ elements is 0 ; thus, $\mathrm{L}$ has class $\mathrm{n}$, which is a contradiction. Thereby, $\mathrm{L}$ can have at most $\mathrm{m}+\mathrm{n}$ generators.

Theorem 11. Let $L$ be a Lie algebra of dimension $r+m$. Suppose each subalgebra of dimension $r$ has class at most $n$. Then the class of $L$ is bounded by

$$
f(m, n)=2^{m} n .
$$

Proof. Since L is a nilpotent Lie algebra, we can find a proper subalgerba of $\mathrm{L}$ that contains $L^{2}$ and has co-dimension 1 . We shall use induction on $\mathrm{m}$. For $\mathrm{m}=1, \operatorname{dim}(L)=\mathrm{r}+1$, and every proper subalgebra of dimension $\mathrm{r}$ has class $\mathrm{n}$ and is an ideal of L.By Lemma 7, L has class at most $n+n=2 n$. 
Suppose the result holds for $\mathrm{m}=\mathrm{k}$. We shall prove that it is also true for $\mathrm{m}=\mathrm{k}+1$. Suppose $\mathrm{m}=\mathrm{k}+1$, and $\operatorname{dim}(L)=\mathrm{r}+\mathrm{k}+1$. We can find two distinct proper subalgebras of $\mathrm{L}$ that have co-dimension 1 . by induction hypothesis, these proper subalgebras have class at most $2^{k} n$. Therefore, L has class at most $2^{k} n+2^{k} n=2^{k+1} n$ by previous lemma.

Hence the class of $\mathrm{L}$ is bounded by $2^{m} n$ for some positive integers $\mathrm{m}$ and n.

\subsection{Some Cases with Small $m$ and $n$}

In this section, we explore the bounds on the nilpotency class in the cases of small $m$ and $n$ other than the classical case $m=n=1$. We assume that a Lie algebra $\mathrm{L}$ has dimension $\mathrm{r}+\mathrm{m}$ and every subalgebra of dimension $\mathrm{r}$ has class $\mathrm{n}$.

Lemma 12. Let $L$ be a nilpotent Lie algebra of dimension $r+1$. Suppose that $L$ is generated by two elements, and every subalgebra of $L$ of dimension $r$ has class at most 2. Then $L$ is metabelian.

Proof. Suppose $\{a, b\}$ is a generating set of $\mathrm{L}$, and we form two distinct maximal subalgebras, $\mathrm{M}$ and $\mathrm{N}$. We have $L=F a+F b+L^{2}, M=F a+L^{2}$, $N=F b+L^{2}$, so

$$
L^{2}=\left[L^{2}, L^{2}\right]+\left[L^{2}, a\right]+\left[L^{2}, b\right]+F[a, b]=M^{2}+N^{2}+F[a, b]
$$

whence

$$
\begin{aligned}
& {\left[L^{2}, L^{2}\right]=0 \text { since }\left[M^{2}, M^{2}\right],\left[M^{2}, N^{2}\right] \subseteq M^{3}=0 .} \\
& {\left[N^{2}, N^{2}\right] \subseteq N^{3}=0 \text { and }[a, b] \in M \cap N}
\end{aligned}
$$

At this point, we are ready to look at class bounds in the cases of small $\mathrm{m}$ and n.

Corollary 13. Let $L$ be a nilpotent Lie algebra over a field of characterisitic not equal to 2. Suppose that $L$ has dimension $r+1$ in which every subalgebra of dimension $r$ has class at most 2. Then $L$ has class at most 3 .

Proof. Observe that $\mathrm{L}$ has class at most 4, and by theorem 15, we may assume that $\mathrm{L}$ is generated by at most three elements. Consider the following possibilities:

Case 1. Suppose no two elements generate L. Then each $(x, y)$ is a proper subalgebra of L that has class 2. Note that L satisfies the 2nd Engel condition; 
that is for any $x, y, z \in L$

$$
\begin{gathered}
0=[x, y+z, y+z] \\
=[x, y, y]+[x, y, z]+[x, z, y]+[x, z, z] \\
=[x, y, z]+[x, z, y] .
\end{gathered}
$$

Therefore, we have the relation that for any $x, y, z \in L$,

$$
[x, y, z]=-[x, z, y]=[z, x, y] .
$$

Now, we want to show that every commutator of length four is zero. Consider the commutator $[a, b ; c, d]$ for any $a, b, c, d \in L$. Using the relation A Bound on the Nilpotency Class in Eq.(5), we have

$$
\begin{gathered}
{[a, b ; c, d]=[c, d, a, b]} \\
=-[c, a, d, b] \\
=[a, c, d, b] \\
=-[a, c, b, d] \\
=[a, b, c, d]
\end{gathered}
$$

But also,

$$
[c, d ; a, b]=-[c,[a, b], d]=[[a, b], c, d]=[a, b, c, d]
$$

As a result of Eqs. (12) and (13)

$$
[a, b, c, d]=[a, b ; c, d]=[c, d ; a, b]=-[a, b ; c, d]=0 .
$$

Thereby, $[a, b, c, d]=0$, and $\mathrm{L}$ has class at most 3 . Note that if the characterstic of the field $\mathrm{F}$ is not equal to 3,then $\mathrm{L}$ has class 2 .

Case 2. Suppose $\mathrm{L}$ is generated by two elements. L is metabelian because of Lemma 17. Therefore, by theorem 9 , the class of $\mathrm{L}$ cannot be precisely 4 , and so is at most 3 .

From the preceding two cases, L has class at most 3.

The next corollary is an immediate consequence of above corollary

Corollary 14. Let $L$ be a nilpotent Lie algebra over a field of characterisitic not equal to 2. Suppose that $L$ has dimension $r+2$ in which every subalgebra of dimension $r$ is abelian. Then $L$ has class at most 3 .

In the following theorem, we consider the case when $\mathrm{m}=\mathrm{n}=2$. 
Theorem 15. Let $L$ be a nilpotent Lie algebra over a field of characteristic not equal to 3. Suppose $L$ has dimension $r+2$, and every subalgebra of dimension $r$ has class at most 2. Then the class of $L$ is at most 4 .

Proof. We know that either $\mathrm{L}$ has class at most 2 or $\mathrm{L}$ has at most 4 generators. If $\mathrm{L}$ has class at most 2 , we are done. Suppose $\mathrm{L}$ has at most 4 generators. Since L is nilpotent, $\operatorname{dim}\left(L / L^{2}\right) \leq 4$. We have to consider all possibilities as follows.

Case 1. Suppose $\operatorname{dim}\left(L / L^{2}\right)=4$.

Every subalgebra generated by two elements is contained in a subalgebra of codimension 2, which has class 2. L satisfies the 2nd Engel condition and has class at most 2 .

Case 2. Let L be generated by 3 elements; that is

$$
\operatorname{dim}\left(L / L^{2}\right)=3 .
$$

Suppose every maximal subalgebra of L has less than three generators. Let

$$
M=(a, b)+L^{2}
$$

be an arbitrary maximal subalgebra of L generated by two elements; hence, $\operatorname{dim}\left(M / M^{2}\right)=2$. Observe that

$$
L \supseteq M \supseteq L^{2} \supseteq M^{2} .
$$

Since $\operatorname{dim}\left(L / L^{2}\right)=3, \operatorname{dim}(L / M)$, and $\operatorname{dim}\left(M / M^{2}\right)=2$ we have that $L^{2}=M^{2}$ for all maximal subalgebras of L. Next consider

$$
M^{2}=([a, b])+M^{3}=L^{2} .
$$

Observe that

$$
L^{2}=M^{2} \supseteq L^{3} \supseteq M^{3}
$$

and $\operatorname{dim}\left(M^{2} / M^{3}\right)=1$; hence, $L^{3}=M^{3}$ for all maximal subalgebras of $\mathrm{L}$. Then, pick another maximal subalgebra, $N=(a, c)+L^{2}$ and look at

$$
L^{2}=M^{2}=([a, b])+M^{3}=([a, b])+L^{3}
$$

and

$$
L^{2}=N^{2}=([a, c])+N^{3}=([a, c])+L^{3} .
$$

Clearly, $[a, b]=\alpha[a, c] \bmod L^{3}$, where $\alpha$ is a scalar. Hence

$$
[a, b-\alpha c] \in L^{3} \text {. }
$$


Suppose $\mathrm{H}$ is the maximal subalgebra such that $H=(a, b-\alpha c)+L^{2}$. Then

$$
L^{2}=H^{2}=([a, b-\alpha c])+H^{3}=([a, b-\alpha c])+L^{3}=L^{3} .
$$

Therefore, $L^{2}=L^{3}=0$, and $\mathrm{L}$ is abelian.

Assume, therefore, that there exist a maximum subalgebra $K$ generated by no pair of its elements; that is $\operatorname{dim}\left(K / K^{2}\right) \geq 3$.For any $x, y \in K$, the subalgebra generated by $\mathrm{x}$ and $\mathrm{y}$ is a proper subalgebra of $\mathrm{K}$. Thus, $\mathrm{x}$ and $\mathrm{y}$ are contained in a maximal subalgebra $\mathrm{P}$ of $\mathrm{K}$. Then $\operatorname{dim}(P)=\operatorname{dim}(K)-1=\operatorname{dim}(L)-2$. Since $\mathrm{P}$ is a subalgebra of $\mathrm{L}$ with co-dimension $2, \mathrm{P}$ has class at most 2 . $\mathrm{K}$ satisfies the 2nd Engel condition which is

$$
[x, y, y]=0
$$

for any $x, y \in K$. Hence, $\mathrm{K}$ has class at most 2. We then consider $L=\langle z\rangle+K$ for some $z \notin K$. Because $\langle z\rangle \subseteq(z)+L^{2}$ anddim $\left(L / L^{2}\right)=3$, we can say that

$$
\operatorname{dim}(\langle z\rangle) \leq \operatorname{dim}(L)-2 .
$$

Therefore. L has class at most 4.

Case 3. We are left with the final case in which $\mathrm{L}$ is generated by 2 elements.

Suppose $\mathrm{L}$ is generated by $\{\mathrm{a}, \mathrm{b}\}$ and $\operatorname{dim}\left(L / L^{2}\right)=2$. Then the algebra generated by $\mathrm{x}$ and $L^{3}$ for any $x \in L$ has class 2 . Thus, we have the identity

$$
[r, s, t, x, x]=0 \text {. }
$$

Since $\mathrm{L}$ is generated by $\{a, b\}$, to show that $\mathrm{L}$ has class at most 4 , it suffices to show that each of the commutators of length 5 that contains $\mathrm{a}$ and $\mathrm{b}$ is 0 . Considering commutators of length 5 , we find that

$$
[a, b, a, a+b, a+b]=0[a, b, a, a, b]=-[a, b, a, b, a]
$$

then

$$
0=[a, b ; a, b]=[a, b, a, b]+[a ; a, b, b] .
$$

As a result, we have

$$
[a, b, a, b]=[a, b, b, a]
$$

for any $a, b \in L$. From both facts in Eqs.(15) and (17), we find

$$
[a, b, a, a, b]=-[a, b, a, b, a]=-[a, b, b, a, a]=0
$$

The fact in Eq. (18) is enough to establish that L has class at most 4. Thus, the proof is complete. 


\section{References}

[1] D. W. Barnes, Nilpotency of Lie algebra, Math. Zeitschrift 79 (1962), 237-238.

[2] N. Jacobson, Lie Algebras, New York, Dover, (1962).

[3] I. D. Macdonald, On certain varieties of groups, Math. Zeitchrift 76, (1961) 270-282.

[4] I. D. Macdonald, Generalisations of a classical theorem about nilpotent groups, Illinois J. Math. 8 , (1964) 556-570. 
